BULL. AUSTRAL. MATH. SOC.

VOL. $23(1981), 139-142$.

\title{
STABILITY OF STRONGLY REGULAR GRAPHS
}

\author{
S.K. Shukla, M.R. SRidharan and S.P. Mohanty
}

In this note we characterize strongly regular graphs which are stable.

\section{Introduction}

Transposition in the automorphism group of a graph is a necessary condition for a graph to be stable, but sufficiency conditions for stability of graphs have not yet been found. However, Holton [2] has shown that a tree is stable if and only if it contains a transposition in its automorphism group. Here we prove a similar result for strongly regular graphs.

We refer to [1] for the definitions and results not mentioned here. For a vertex $u$ of a graph $G$, by $N_{G}(u)$ we denote the set of all vertices adjacent to $u$. By $\overline{N_{G}(u)}$, we denote the set $N_{G}(u) \cup\{u\}$.

If $W \subseteq V(G)$, then by $G_{W}$ we mean the induced subgraph $(V(G)-W\rangle$ of $G$. By $\Gamma(G)_{W}$, we denote the maximal subgroup of $\Gamma(G)$ each element of which fixes each vertex in $W$; here we consider $\Gamma(G)_{W}$ as acting only on $V(G)-W$. A graph $G$ is said to be semi-stable (at $v \in V(G)$ ) if $\Gamma\left(G_{v}\right)=\Gamma(G)_{v}$. If there exists a sequence $v_{1}, v_{2}, \ldots, v_{n}$ of all the vertices of $G$ such that $G_{\left\{v_{1}, \ldots, v_{k}\right\}}$ is semi-stable at $v_{k+1}$ for $1 \leq k \leq n-1$, we say that $G$ is stable.

Received 9 September 1980. 
An $r$-regular graph $G$ of order $n$ is said to be strongly regular if

(i) the number of vertices adjacent to both end vertices of an edge is constant and is equal to $\lambda$,

(ii) the number of vertices adjacent to two non-adjacent vertices is constant and is equal to $\mu$.

$n, r, \lambda$ and $\mu$ are called the parameters of $G$.

Now we list the following results which we require to prove the main result.

LEMMA 1 [3]. If a graph $G$ is stable, then either $G$ is $K_{1}$ or $\Gamma(G)$ contains a transposition.

LEMMA 2 [3]. If a graph $G$ is stable then $\bar{G}$ is stable.

LEMMA 3 [4]. If $G$ is a strongly regular graph with parameters $n$, $r, \lambda$ and $\mu$, then $\bar{G}$ is a strongly regular graph with parameters $\bar{n}, \bar{r}$, $\bar{\lambda}$, and $\bar{\mu}$ where $\bar{n}=n, \bar{r}=n-r-1, \bar{\lambda}=n-2 r+\mu-2$ and $\bar{\mu}=n-2 r+\lambda$.

\section{Main result}

THEOREM 1. A nontrivial strongly-regular graph $G$ with parameters $n, r, \lambda$ and $\mu$ has a transposition in the automorphism group if and only if $G \cong m K_{r}$ or $\overline{m K_{n-r-1}}$ for some $m \geq 1$.

Proof. If $G \cong m K_{r}$ or $\overline{m K_{n-r-1}}$ and is not equal to $K_{1}$, then certainly it has a transposition.

Suppose $G$ is strongly-regular and $\Gamma(G)$ contains a transposition $(u v)$. If $[u, v] \in E(G)$, then we prove that $G \cong m K_{r}$. Otherwise we prove that $G \cong \overline{m K_{n-r-1}}$.

$$
\text { Let }[u, v] \in E(G) \text {. Since }(u v) \in \Gamma(G) \text {, }
$$

$$
\overline{N_{G}(u)}=\overline{N_{G}(v)}
$$

or

$$
\overline{N_{G}(u)}-\{u, v\}=\overline{N_{G}(v)}-\{u, v\}
$$


But

$$
d(u)=d(v)=r
$$

therefore $\lambda=r-1$.

Let $w \in \overline{N_{G}(u)}$. Since $[u, w] \in E(G)$ and $\lambda=r-1$, $\overline{N_{G}(u)}=\overline{N_{G}(w)}$. Thus $\overline{N_{G}(u)}$ induces a complete graph of order $r$ in $G$. If $n>r$, then choose any vertex $x \notin \overline{N_{G}(u)}$. Since $G$ is strongly regular with $\lambda=r-1, \overline{N_{G}(x)}$ also induces a graph isomorphic to $K_{r}$ in $G$. If still there is any vertex $y \notin \overline{N_{G}(u)} \cup \overline{N_{G}(x)}$, then $\overline{N_{G}(y)}$ will induce a graph isomorphic to $K_{r}$. Proceeding in this way, till we exhaust all vertices of $G$, we find that every component of $G$ is isomorphic to $K_{r}$, that is, $G=m K_{r}$ for some $m \geq 1$.

If $[u, v] \notin E(G)$ then $[u, v] \in E(\bar{G})$. Since $(u v) \in \Gamma(G)$, $(u v) \in \Gamma(\bar{G})$. From Lemma 3 , we infer that $\bar{G}$ is strongly regular with parameters $\bar{n}, \bar{r}, \bar{\lambda}$, and $\bar{\mu}$ such that $\bar{n}=n, \bar{r}=n-r-1$, $\bar{\lambda}=n-2 r+\mu-2$ and $\vec{\mu}=n-2 r+\lambda$. Since $[u, v] \notin E(G)$,

$$
N_{G}(u)=N_{G}(v) \text {. }
$$

Hence

$$
\mu=\left|N_{G}(u)\right|=r .
$$

Therefore

$$
\bar{\lambda}=n-r-2 .
$$

Since $\bar{G}$ is a strongly regular graph such that $[u, v] \in E(\bar{G})$, $(u v) \in \Gamma(\bar{G})$ and $\bar{\lambda}$ is equal to $n-r-2$, it follows from earlier discussions that $\bar{G} \cong m K_{n-r-1}$ for some $m \geq 1$. Therefore $G \cong \overline{m K_{n-r-1}}$. This completes the proof.

COROLLARY 1. A non-trivial strongly-regular graph is stable if and only if it contains a transposition in the automorphism group.

Proof. Let $G$ be a strongly-regular graph with parameters $n, r, \lambda$ and $\mu$. If $\Gamma(G)$ contains a transposition, it follows from Theorem 1 that $G \cong m K_{r}$ or $\overline{m K_{n-r-1}}$. Since complete graphs are stable, $m K_{p}$ and 
$m K_{n-r-1}$ are also stable. From Lemma 2, we conclude that $\overline{m K_{n-r-1}}$ is stable. Therefore $G$ is stable.

If $G$ is stable, it is clear from Lemma 2 that $\Gamma(G)$ contains a transposition. This completes the proof.

\section{References}

[1] Frank Harary, Graph theory (Addison-Wesley, Reading, Massachusetts; Menlo Park, California; London; 1972).

[2] D.A. Holton, "Stable trees", J. Austral. Math. Soc. 15 (1973), 476-481.

[3] D.A. Holton and Douglas D. Grant, "Regular graphs and stability", $J$. Austral. Math. Soc. Ser. A 20 (1975), 377-384.

[4] Xavier L. Hubaut, "Strongly regular graphs", Discrete Math. 13 (1975), $357-381$.

Department of Mathematics,

Indian Institute of Technology,

Kanpur 208016,

India. 